\title{
Periodic successive approximations and interval halving
}

\author{
A. Rontó and M. Rontó
}




\title{
PERIODIC SUCCESSIVE APPROXIMATIONS AND INTERVAL HALVING
}

\author{
A. RONTÓ AND M. RONTÓ
}

Received 6 December, 2011

\begin{abstract}
We show how a suitable interval halving and parametrisation technique can help to essentially improve the sufficient convergence condition for the periodic successive approximations dealing with periodic solutions of nonlinear non-autonomous systems of ordinary differential equations.
\end{abstract}

2000 Mathematics Subject Classification: 34B15

Keywords: Periodic boundary value problems, parametrisation technique, periodic successive approximations, interval halving

\section{INTRODUCTION}

It is well-known that, formally, the methods used in the the theory of boundary value problems for the investigation and approximate construction of solutions, can be characterised as analytic, functional-analytic, numerical and numerical-analytic ones (see, e. g. [1, 4, 7, 16, 20,23] and the references therein). Among the numericalanalytic methods we share out those which are based upon some types of successive approximations.

In the theory of nonlinear oscillations, such types of numerical-analytic methods were apparently first developed by Cesari [2], Hale [6], and Samoilenko [24-26]. The latter mentioned approach had later been developed for more general types of boundary-value problems in numerous books and papers (see, e. g., [8-14, 17, 21,22, 22, 27-29]).

Methods of the numerical-analytic type, in a sense, combine, advantages of the mentioned above approaches and are usually based upon certain iteration processes constructed explicitly in analytic form. Such an approach belongs to the few of them that offer constructive possibilities both for the investigation of the existence of solution and it approximate construction.

For a boundary value problem, the numerical-analytic approach usually replaces the problem by the Cauchy problem for a suitably perturbed system containing some artificially introduced vector parameter $z$, which most often has the meaning of the 
initial value of the solution and the numerical value of which is to be determined later. A solution of the Cauchy problem for the perturbed system is sought for in an analytic form by successive approximations. The functional "perturbation term," by which the modified equation differs from the original one, depends explicitly on the parameter $z$ and generates a system of algebraic or transcendental "determining equations" from which the numerical values of $z$ should be found. The solvability of the determining system, in turn, may be checked by studying some approximations constructed explicitly. It is clear that the complexity of the given equations and boundary conditions has essential influence on both the efficiency of construction of approximate solutions and the subsequent solvability analysis.

In order to guarantee the convergence, one usually assumes [11,27-29] a kind of the Lipschitz condition and requires a certain smallness restriction, which most often has the form

$$
r(K) \leq q_{T},
$$

where $K$ is the Lipschitz matrix and $q_{T}$ is a constant depending on the period $T$. The improvement of the condition then consists in minimising the value of $q_{T}$.

The aim of this paper is to show how a suitable interval halving and parametrization techniques can, under fairly general assumptions, weaken (1.1) to

$$
r(K) \leq 2 q_{T}
$$

and, thus, essentially improve the sufficent convergence conditions established in [11, 24-27] for the periodic successive approximations related to the periodic solutions of non-linear non-autonomous systems of ordinary differential equations.

\section{LIST OF SYMBOLS}

We fix an $n \in \mathbb{N}$ and a bounded set $D \subset \mathbb{R}^{n}$. The following symbols and conventions are used in the sequel:

(1) $|\cdot|, \leq$ for vectors from $\mathbb{R}^{n}$ are understood component-wise.

(2) $\sup \{f(z): z \in Q\}$ for any $f=\left(f_{i}\right)_{i=1}^{n}: Q \rightarrow \mathbb{R}^{n}$, where $Q \subset \mathbb{R}^{m}, m \leq n$, is defined as the column vector with components $\sup \left\{f_{i}(z): z \in Q\right\}, i=$ $1,2, \ldots, n$. The symbol $\max \{f(z): z \in Q\}$ is defined by analogy.

(3) The "vector-valued diameter" of a set $Q \subset \mathbb{R}^{n}$ is defined by the equality

$$
\operatorname{diam} Q:=\sup \left\{\left|z_{1}-z_{2}\right|:\left\{z_{1}, z_{2}\right\} \subset Q\right\} .
$$

(4) $d(Q)$ : see (7.26).

(5) $r(K)$ is the maximal, in modulus, eigenvalue of a matrix $K$

(6) $\delta_{J, D}(f)$ : see $(4.2)$.

(7) $\varrho_{*}$ : see (4.3) and (4.4).

(8) $D(r)$ : see Definition 4.1, formula (4.1).

(9) $Q-Q:=\left\{z_{1}-z_{2}:\left\{z_{1}, z_{2}\right\} \subset Q\right\}$ for any $Q \subset \mathbb{R}^{n}$. 


\section{Motivation, PROBLEM SETTING, AND BASIC ASSUMPTIONS}

In the papers [24,25], Samoilenko had suggested a method for studying $T$-periodic solutions of a system of non-linear ordinary differential equations

$$
u^{\prime}(t)=f(t, u(t)), \quad t \in(-\infty, \infty),
$$

where $f: \mathbb{R} \times D \rightarrow \mathbb{R}^{n}$ is a continuous function such that

$$
f(t, z)=f(t+T, z)
$$

for all $z \in D$ and all $t \in(-\infty, \infty)$. Here, $T$ is a given positive number and $D$ is the closure of a bounded and connected domain in $\mathbb{R}^{n}$.

We are interested in continuously differentiable solutions of system (3.1) which are periodic with period $T$. From now on, instead $T$-periodic solutions of (3.1), we shall deal with solutions of the corresponding periodic boundary value problem on the bounded interval $[0, T]$

$$
\begin{gathered}
u^{\prime}(t)=f(t, u(t)), \quad t \in[0, T], \\
u(0)=u(T) .
\end{gathered}
$$

The passage to problem (3.3), (3.4) is justified by assumption (3.2) and the following standard lemma (see, e. g., [11, Lemma 3.1] or [3, Lemma 2.2.1]) which is given here for completeness.

Lemma 3.1. If a function $u:(-\infty, \infty) \rightarrow D$ is a $T$-periodic solution of a system (3.1), then its restriction to $[0, T]$ satisfies (3.3), (3.4). Conversely, if, under assumption (3.2), a function $u:[0, T] \rightarrow D$ is a solution of the boundary value problem (3.3), (3.4), then its continuous $T$-periodic extension to $(-\infty, \infty)$ is a T-periodic solution of system (3.1).

Our study of the periodic boundary value problem (3.3), (3.4) is based on the assumption that the non-linearity $f:[0, T] \times D \rightarrow \mathbb{R}^{n}$ is Lipschitzian in the space variable. For the sake of simplicity, we assume that there exists a non-negative constant matrix $K=\left(k_{i j}\right)_{i, j=1}^{n}$ such that

$$
\left|f\left(t, x_{1}\right)-f\left(t, x_{2}\right)\right| \leq K\left|x_{1}-x_{2}\right|
$$

for all $\left\{x_{1}, x_{2}\right\} \subset D$ and $t \in[0, T]$.

Here and below, the obvious notation $|x|=\operatorname{col}\left(\left|x_{1}\right|,\left|x_{2}\right|, \ldots,\left|x_{n}\right|\right)$ is used. The inequalities and the signs "max" and "min" for vectors are understood componentwise.

The method of $[24,25]$, originally called numerical-analytic method for the investigation of periodic solutions, was later referred to also as numerical-analytic method of periodic successive approximations [11,27-29].

The scheme of the method, which is described, in a suitable for us form, by Propositions 5.1 and 5.5 below, is extremely simple and deals with the investigation of the 
parametrised equation

$$
u(t)=z+\int_{0}^{t} f(s, u(s)) d s-\frac{t}{T} \int_{0}^{T} f(s, u(s)) d s, \quad t \in[0, T],
$$

where $z \in D$ is an parameter to be chosen later. For convenience of reference, we formulate the statements for the $p$-periodic problem

$$
\begin{gathered}
u^{\prime}(t)=g(t, u(t)), \quad t \in\left[t_{0}, t_{0}+p\right], \\
u\left(t_{0}\right)=u\left(t_{0}+p\right),
\end{gathered}
$$

where $g:\left[t_{0}, t_{0}+p\right] \times \mathbb{R}^{n} \rightarrow \mathbb{R}^{n}$ and $t_{0} \in(-\infty, \infty)$ is arbitrary but fixed.

\section{NOTATION}

The notion of a set $D(r)$ associated with $D$, which could have been called an $r$-core of $D$, will often be used in the sequel.

Definition 4.1. For any non-negative vector $r \in \mathbb{R}^{n}$, we put

$$
D(r):=\{z \in D: B(z, r) \subset D\},
$$

where $B(z, r):=\left\{\xi \in \mathbb{R}^{n}:|\xi-z| \leq r\right\}$.

Definition 4.2. Given a closed interval $J \subseteq \mathbb{R}$, define the vector

$$
\delta_{J, D}(f):=\max _{(t, z) \in J \times D} f(t, z)-\min _{(t, z) \in J \times D} f(t, z) .
$$

Finally, let the positive number $\varrho_{*}$ be determined by the equality

$$
\varrho_{*}^{-1}=\inf \left\{q>0: q^{-1}=\int_{0}^{\frac{1}{2}} \exp (\tau(\tau-1) q) d \tau\right\} .
$$

Note that the constant $\varrho_{*}$ had already appeared in the literature, and it can be defined in various ways (see, e. g., $[11,16]$ for more details). One can show that

$$
\varrho_{*} \approx 0.2927 \text {. }
$$

\section{5. $p$-PERIODIC SUCCESSIVE APPROXIMATIONS: THE ORIGINAL VERSION}

The original, unmodified, periodic successive approximations scheme for the $p$ periodic problem (3.7), (3.8) is constructed as follows. With problem (3.7), (3.8), we associate the sequence of functions $u_{m}(\cdot, z), m \geq 0$, defined according to the rule

$$
\begin{aligned}
u_{0}(t, z) & :=z \\
u_{m}(t, z) & :=z+\int_{t_{0}}^{t} g\left(s, u_{m-1}(s, z)\right) d s-\frac{t-t_{0}}{p} \int_{t_{0}}^{t_{0}+p} g\left(s, u_{m-1}(s, z)\right) d s
\end{aligned}
$$

for $t \in\left[t_{0}, t_{0}+p\right]$ and $m=1,2, \ldots$, where the vector $z=\operatorname{col}\left(z_{1}, z_{2}, \ldots, z_{n}\right)$ is regarded as a parameter whose value is to be determined later. The following proposition is a consequence of [8, Theorem 1]. 
Proposition 5.1 ([8]). Let the function g satisfy the Lipschitz condition of form (3.5) with a matrix $K$ for which the inequality

$$
r(K)<\frac{1}{p \varrho_{*}}
$$

holds, and moreover

$$
D\left(\frac{p}{4} \delta_{\left[t_{0}, t_{0}+p\right], D}(g)\right) \neq \varnothing .
$$

Then, for any fixed $z \in D\left(\frac{p}{4} \delta_{\left[t_{0}, t_{0}+p\right], D}(g)\right)$, the following assertions are true:

(1) Sequence (5.1) converges to a limit function

$$
u_{\infty}(t, z)=\lim _{m \rightarrow \infty} u_{m}(t, z)
$$

uniformly in $t \in\left[t_{0}, t_{0}+p\right]$.

(2) The limit function (5.4) satisfies p-periodic boundary conditions

$$
u_{\infty}\left(t_{0}, z\right)=u_{\infty}\left(t_{0}+p, z\right) .
$$

(3) The function $u_{\infty}(\cdot, z)$ is the unique solution of Cauchy problem

$$
\begin{gathered}
u^{\prime}(t)=g(t, u(t))-p^{-1} \Delta(z), \quad t \in\left[t_{0}, t_{0}+p\right], \\
u\left(t_{0}\right)=z,
\end{gathered}
$$

where

$$
\Delta(z):=\int_{t_{0}}^{t_{0}+p} g\left(\tau, u_{\infty}(\tau, z)\right) d \tau .
$$

(4) Given an arbitrarily small positive $\varepsilon$, one can choose a number $m_{\varepsilon} \geq 1$ such that the estimate

$$
\left|u_{m}(t, z)-u_{\infty}(t, z)\right| \leq \frac{\alpha_{m_{\varepsilon}}(t)}{2} K^{m_{\varepsilon}-1}\left(p c_{\varepsilon} K\right)^{m-m_{\varepsilon}+1}\left(1_{n}-p c_{\varepsilon} K\right)^{-1} \delta_{\left[t_{0}, t_{0}+p\right], D}(g)
$$

holds for all $t \in\left[t_{0}, t_{0}+p\right]$ and $m \geq m_{\varepsilon}$, where

$$
c_{\varepsilon}:=\varrho_{*}+\varepsilon .
$$

Remark 5.2. According to Definition 4.1, condition (5.3) means the non-emptiness of the $\frac{p}{4} \delta_{\left[t_{0}, t_{0}+p\right], D}(g)$-core of the set $D$, where the vector $\delta_{\left[t_{0}, t_{0}+p\right], D}(g)$ is given by formula (4.2). This correlates with the natural idea that the domain where the Lipschitz condition is assumed should be wide enough.

The proof of Proposition 5.1 is based on Lemma 5.3 formulated below, which provides an estimate for the sequence of functions $\alpha_{m}, m \geq 0$, given by the formula

$$
\alpha_{m}(t):=\left(1-\frac{t-t_{0}}{p}\right) \int_{t_{0}}^{t} \alpha_{m-1}(s) d s+\frac{t-t_{0}}{p} \int_{t}^{t_{0}+p} \alpha_{m-1}(s) d s
$$

where $\alpha_{0}(t):=1, t \in\left[t_{0}, t_{0}+p\right], m \geq 1$. 
Lemma 5.3. Given an arbitrary $\varepsilon \in(0,+\infty)$, one can specify an integer $m_{\varepsilon} \geq 1$ such that

$$
\alpha_{m+1}(t) \leq p\left(\varrho_{*}+\varepsilon\right) \alpha_{m}(t)
$$

for all $t \in\left[t_{0}, t_{0}+p\right]$ and $m \geq m_{\varepsilon}$, where $\varrho_{*} \approx 0.2927$ is given by (4.3).

Proof. The assertion is easily derived from [8, Lemma 3] by transforming the time interval to $\left[t_{0}, t_{0}+p\right]$.

Remark 5.4. It follows from [19, Lemma 4] that if $\varepsilon \geq \varepsilon_{0}$, where

$$
\varepsilon_{0}:=\frac{3}{10}-\varrho_{*} \approx 0.00727
$$

then $m_{\varepsilon}=1$ in Lemma 5.3.

Proposition 5.1 suggests that there is a relation between $p$-periodic solutions of the given differential equation and those of the perturbed equation (5.5), which are, at the same time, solutions of the initial value problem (5.5), (5.6). The following statement shows that, by choosing the value of $z$ appropriately, one can use function (5.4) to construct a solution of the original periodic problem (3.3), (3.4).

Proposition 5.5. Let the assumptions of Proposition 5.1 hold. Then:

(1) Given a $z \in D\left(\frac{p}{4} \delta_{\left[t_{0}, t_{0}+p\right], D}(g)\right)$, the function $u_{\infty}(\cdot, z)$ is a solution of the p-periodic boundary value problem (3.7), (3.8) if and only if $z$ is a root of the equation

$$
\Delta(z)=0 .
$$

(2) For any solution $u(\cdot)$ of problem (3.7), (3.8) with $u\left(t_{0}\right) \in D\left(\frac{p}{4} \delta_{\left[t_{0}, t_{0}+p\right], D}(g)\right)$, there exists $a z_{0}$ such that $u(\cdot)=u_{\infty}\left(\cdot, z_{0}\right)$.

The important assertion (2) means that equation (5.12), usually referred to as a determining equation, allows one to track all the solutions of the periodic boundary value problem (3.3), (3.4). The original infinite-dimensional problem is thus reduced to a system of $n$ numerical equations.

Proof of Proposition 5.5. The statement is a consequence of [8, Corollary 1] and [15, Lemma 3.5].

The method thus consists of two parts, namely, the analytic part, when the integral equation (3.6) is dealt with by using the method of successive approximations (5.1), and the numerical one which consists in finding a value of the unknown parameter from equation (5.12).

We also note that it is possible to prove the existence of a solution based on the properties of a certain iteration $u_{m}(\cdot, z)$, constructed explicitly for a certain fixed $m$, by studying the approximate determining system

$$
\Delta_{m}(z)=0
$$


where $\Delta_{m}: D \rightarrow \mathbb{R}^{n}$ is defined by the formula

$$
\Delta_{m}(z):=\int_{t_{0}}^{t_{0}+p} g\left(s, u_{m}(s, z)\right) d s, \quad z \in D .
$$

This topic is discussed in detail, in particular, in [11], whereas a theorem of the kind specified, which corresponds to the scheme developed here, is proved in Section 10 below.

In view of (4.4), assumption (5.2), which is essential for the proof of the uniform convergence of sequence (5.1), can be rewritten in the form

$$
\operatorname{pr}(K)<3.4161 \ldots
$$

Inequality (5.13) can be treated either as a kind of upper bound for the Lipschitz matrix or as a smallness assumption on the period $p$, the latter interpretation presenting the scheme as particularly appropriate for the study of high-frequency oscillations.

Without assumption (5.13), Lemma 5.3 does not guarantee the convergence of sequence (5.1) directly. Nevertheless, it turns out that this limitation can be overcome. We are going to show that, by using a suitable parametrisation and modifying the scheme appropriately, one can always weaken the smallness condition (5.2) so that the constant on its right-hand side is doubled:

$$
r(K)<\frac{2}{p \varrho_{*}} .
$$

\section{INTERVAL HALVING AND PARAMETRISATION}

Our aim is to show that the approach described above can also be used in the cases where the smallness condition (5.2), which guarantees the convergence, is not satisfied. For this purpose, a natural trick based on the interval halving can be used.

The idea is surprisingly simple: along with the periodic boundary value problem (3.3), (3.4), consider two auxiliary problems,

$$
\begin{gathered}
x^{\prime}(t)=f(t, x(t)), \quad t \in\left[0, \frac{1}{2} T\right], \\
x\left(\frac{T}{2}\right)-x(0)=\lambda
\end{gathered}
$$

and

$$
\begin{gathered}
y^{\prime}(t)=f(t, y(t)), \quad t \in\left[\frac{1}{2} T, T\right], \\
y(T)-y\left(\frac{T}{2}\right)=-\lambda,
\end{gathered}
$$

where $\lambda=\operatorname{col}\left(\lambda_{1}, \ldots, \lambda_{n}\right)$ is a parameter. Our further reasoning related to problem (3.3), (3.4) uses the following simple observation. 
Proposition 6.1. Let $x:\left[0, \frac{1}{2} T\right] \rightarrow \mathbb{R}^{n}$ and $y:\left[\frac{1}{2} T, T\right] \rightarrow \mathbb{R}^{n}$ be solutions of problems (6.1), (6.2) and (6.3), (6.4), respectively, with a certain value of $\lambda \in \mathbb{R}^{n}$. Then the function

$$
u(t):=\chi_{T}(t) x(t)+\left(1-\chi_{T}(t)\right)\left(y(t)-y\left(\frac{T}{2}\right)+x\left(\frac{T}{2}\right)\right), \quad t \in[0, T],
$$

where $\chi_{T}(t)=1$ for $0 \leq t<\frac{1}{2} T$ and $\chi_{T}(t)=0$ for $\frac{1}{2} T \leq t \leq T$, is a solution of the periodic problem boundary value problem (3.4) for the equation

$$
u^{\prime}(t)=f\left(t, u(t)+\left(1-\chi_{T}(t)\right)\left(y\left(\frac{T}{2}\right)-x\left(\frac{T}{2}\right)\right)\right), \quad t \in[0, T] .
$$

Conversely, if a certain function $u:[0, T] \rightarrow \mathbb{R}^{n}$ is a solution of problem (3.3), (3.4), then its restrictions $x:=\left.u\right|_{\left[0, \frac{1}{2} T\right]}$ and $y:=\left.u\right|_{\left[\frac{1}{2} T, T\right]}$ to the corresponding intervals satisfy, respectively, problems (6.1), (6.2) and (6.3), (6.4).

Proof. The idea is to rewrite the periodic boundary condition (3.4) in the form

$$
u(0)-u\left(\frac{T}{2}\right)+u\left(\frac{T}{2}\right)-u(T)=0 .
$$

Then it is obvious from (6.2) and (6.4) that the function $u$ given by (6.5) satisfies (6.7) and, hence, condition (3.4) holds. Moreover, it is clear from (6.5) that $u\left(\frac{1}{2} T\right)=$ $x\left(\frac{1}{2} T\right)$. Thus, $u$ is continuous at the point $\frac{1}{2} T$ and, hence, $u \in C\left([0, T], \mathbb{R}^{n}\right)$. By direct computation, one easily verifies that function (6.5) satisfies equation (6.6) and, therefore, is a solution of problem (3.4), (6.6).

On the other hand, if a certain function $u:[0, T] \rightarrow \mathbb{R}^{n}$ is a solution of problem (3.3), (3.4), then its restriction $x:=\left.u\right|_{\left[0, \frac{1}{2} T\right]}$ to the interval $\left[0, \frac{1}{2} T\right]$ satisfies (6.2) with

$$
\lambda:=u\left(\frac{T}{2}\right)-u(0) .
$$

Put now $y:=\left.u\right|_{\left[\frac{1}{2} T, T\right]}$. Then, due to (3.4) and (6.8), we have

$$
\begin{aligned}
y(T)-y\left(\frac{T}{2}\right) & =u(0)-u\left(\frac{T}{2}\right) \\
& =-\lambda
\end{aligned}
$$

and, therefore, $y$ has property (6.4) with $\lambda$ given by (6.8).

Proposition 6.1 suggests one the idea to treat the $T$-periodic problem (3.3), (3.4) as a kind of join of two independent two-point problems. More precisely, one may try to solve independently the auxiliary problems (6.1), (6.2) and (6.3), (6.4), considering $\lambda$ as an unknown parameter, and then "glue" their solutions together by choosing the value of $\lambda$ so that (6.9) holds. A rigorous formulation is contained in the following 
Proposition 6.2. Assume that $x:\left[0, \frac{1}{2} T\right] \rightarrow \mathbb{R}^{n}$ and $y:\left[\frac{1}{2} T, T\right] \rightarrow \mathbb{R}^{n}$ are solutions of problems (6.1), (6.2) and (6.3), (6.4), respectively, for a certain value of $\lambda \in \mathbb{R}^{n}$. Then the function $u:[0, T] \rightarrow \mathbb{R}^{n}$ given by formula (6.5) is a solution of problem (3.3), (3.4) if and only if the equality

$$
x\left(\frac{T}{2}\right)=y\left(\frac{T}{2}\right)
$$

holds.

Conversely, if a certain $u:[0, T] \rightarrow \mathbb{R}^{n}$ is a solution of problem (3.3), (3.4), then the functions $x:=\left.u\right|_{\left[0, \frac{1}{2} T\right]}$ and $y:=\left.u\right|_{\left[\frac{1}{2} T, T\right]}$ satisfy, respectively, problems (6.1), (6.2) and (6.3), (6.4).

Proof. It is sufficient to apply Proposition 6.1 and take into account the fact that, for $x:\left[0, \frac{1}{2} T\right] \rightarrow \mathbb{R}^{n}$ and $y:\left[\frac{1}{2} T, T\right] \rightarrow \mathbb{R}^{n}$ satisfying (6.9), equality (6.6) coincides with (3.3).

The second part of the assertion is a repetition of that of Proposition 6.1. Note that condition (6.9) is satisfied automatically in that case.

\section{SUCCESSIVE APPROXIMATIONS ON SMALLER INTERVALS}

Let us now turn to the construction of the successive approximation scheme related to the $T$-periodic problem (3.3), (3.4). As Proposition 6.2 suggests, we shall first deal with the auxiliary problems (6.1), (6.2) and (6.3), (6.4), for which purpose appropriate iteration processes will be introduced.

More precisely, for any $\xi \in \mathbb{R}^{n}$ and $\lambda \in \mathbb{R}^{n}$, we set

$$
x_{0}(t, \xi, \lambda):=\xi+\frac{2 t}{T} \lambda, \quad t \in\left[0, \frac{1}{2} T\right]
$$

and define the recurrence sequence of functions $x_{m}:\left[0, \frac{1}{2} T\right] \times \mathbb{R}^{2 n} \rightarrow \mathbb{R}^{n}, m=$ $0,1, \ldots$, by putting

$$
\begin{aligned}
x_{m}(t, \xi, \lambda):= & \int_{0}^{t} f\left(s, x_{m-1}(s, \xi, \lambda)\right) d s-\frac{2 t}{T} \int_{0}^{\frac{T}{2}} f\left(s, x_{m-1}(s, \xi, \lambda)\right) d s \\
& +\xi+\frac{2 t}{T} \lambda, \quad t \in\left[0, \frac{1}{2} T\right],
\end{aligned}
$$

for all $m=1,2, \ldots$.

Introduce the functions $\bar{\alpha}_{m}:\left[0, \frac{1}{2} T\right] \rightarrow[0,+\infty)$ and $\overline{\bar{\alpha}}_{m}:\left[\frac{1}{2} T, T\right] \rightarrow[0,+\infty)$, $m \geq 0$, by putting $\bar{\alpha}_{0} \equiv 1, \overline{\bar{\alpha}}_{0} \equiv 1$,

$$
\bar{\alpha}_{m+1}(t):=\left(1-\frac{2 t}{T}\right) \int_{0}^{t} \bar{\alpha}_{m}(s) d s+\frac{2 t}{T} \int_{t}^{\frac{1}{2} T} \bar{\alpha}_{m}(s) d s
$$


for $t \in\left[0, \frac{1}{2} T\right]$, and

$$
\overline{\bar{\alpha}}_{m+1}(t):=2\left(1-\frac{t}{T}\right) \int_{\frac{1}{2} T}^{t} \overline{\bar{\alpha}}_{m}(s) d s+\left(\frac{2 t}{T}-1\right) \int_{t}^{T} \overline{\bar{\alpha}}_{m}(s) d s
$$

for $t \in\left[\frac{1}{2} T, T\right]$. In particular, we have

$$
\bar{\alpha}_{1}(t)=2 t\left(1-\frac{2 t}{T}\right), \quad t \in\left[0, \frac{1}{2} T\right]
$$

and

$$
\overline{\bar{\alpha}}_{1}(t)=2\left(1-\frac{t}{T}\right)(2 t-T), \quad t \in\left[\frac{1}{2} T, T\right] .
$$

Functions (7.3) and (7.4) are involved in the estimates of convergence given in the sequel. In the case of the iteration process (7.2), we have the following

Theorem 7.1. Let the vector-function $f:[0, T] \times D \rightarrow \mathbb{R}^{n}$ satisfy the Lipschitz condition (3.5) on the set $D$ with a matrix $K$ such that

$$
r(K)<\frac{2}{T \varrho_{*}},
$$

where the constant $\varrho_{*}$ is determined by equality (4.3). Moreover, assume that there exists a set $\mathscr{D} \subset D$ such that

$$
D\left(\frac{T}{8} \delta_{\left[0, \frac{1}{2} T\right], D}(f)+\operatorname{diam} \mathscr{D}\right) \neq \varnothing .
$$

Then, for all $\lambda \in \mathscr{D}-\mathscr{D}$ and $\xi \in D\left(\frac{T}{8} \delta_{\left[0, \frac{1}{2} T\right], D}(f)+\operatorname{diam} \mathscr{D}\right)$ :

(1) For any $m \geq 1, x_{m}(\cdot, \xi, \lambda)$ satisfies the boundary conditions

$$
x_{m}\left(\frac{1}{2} T, \xi, \lambda\right)-x_{m}(0, \xi, \lambda)=\lambda .
$$

(2) The uniform, in $t \in\left[0, \frac{1}{2} T\right]$, limit

$$
\lim _{m \rightarrow \infty} x_{m}(t, \xi, \lambda)=: x_{\infty}(t, \xi, \lambda)
$$

exists, and moreover,

$$
x_{\infty}\left(\frac{1}{2} T, \xi, \lambda\right)-x_{\infty}(0, \xi, \lambda)=\lambda
$$

(3) The function $x_{\infty}(\cdot, \xi, \lambda)$ is the unique solution of the Cauchy problem

$$
\begin{gathered}
x^{\prime}(t)=f(t, x(t))+2 T^{-1} \Xi(\xi, \lambda), \\
x(0)=\xi,
\end{gathered}
$$

where

$$
\Xi(\xi, \lambda):=\lambda-\int_{0}^{\frac{T}{2}} f\left(\tau, x_{\infty}(\tau, \xi, \lambda)\right) d \tau
$$


(4) For an arbitrarily small positive $\varepsilon$, one can find a number $m_{\varepsilon} \geq 1$ such that the function

$$
\xi_{m}(\cdot, \xi, \lambda):=x_{m}(\cdot, \xi, \lambda)-x_{\infty}(\cdot, \xi, \lambda)
$$

satisfies the estimate

$$
\left|\xi_{m}(t, \xi, \lambda)\right| \leq \frac{1}{2} \bar{\alpha}_{m_{\varepsilon}}(t) K^{m_{\varepsilon}-1}\left(\frac{1}{2} T c_{\varepsilon} K\right)^{m-m_{\varepsilon}+1}\left(1_{n}-\frac{1}{2} T c_{\varepsilon} K\right)^{-1} \delta_{\left[0, \frac{1}{2} T\right], D}(f)
$$

for all $t \in\left[0, \frac{1}{2} T\right]$ and $m \geq m_{\varepsilon}$, where $c_{\varepsilon}$ is given by (5.8).

Recall that the non-negative vector diam $\mathscr{D}$ appearing in condition (7.8) is defined according to formula (2.1). By $\mathscr{D}-\mathscr{D}$, we mean the set of all the differences $z_{1}-z_{2}$ with $z_{1}$ and $z_{2}$ both belonging to $\mathscr{D}$. The proof of Theorem 7.1 is postponed till Section 8.4 .

Remark 7.2. The error estimate (7.15) may look inconvenient because it is guaranteed starting from a sufficiently large iteration number, $m_{\varepsilon}$, depending on the value of $\varepsilon$ which can be arbitrarily small. It is, however, quite transparent when the required constant is not "too close" to $\varrho_{*}$ (i. e., if $\varepsilon$ is not "too small").

More precisely, as is seen from the proof given in Section $8, m_{\varepsilon}$ in Theorem 7.1 is, in fact, the very number appearing in Lemma 5.3. In view of Remark 5.4, $m_{\varepsilon}=1$ for $\varepsilon \geq \varepsilon_{0}$, where

$$
\varepsilon_{0} \approx 0.00727
$$

is given by formula (5.11). Consequently, inequality (7.15) with $\varepsilon \geq \varepsilon_{0}$ holds for an arbitrary value of $m \geq 1$.

By analogy to Theorem 7.1, let us now consider the parametrised boundary value problem (6.3), (6.4) on the interval $\left[\frac{1}{2} T, T\right]$. Put

$$
y_{0}(t, \eta, \lambda):=\eta-\left(\frac{2 t}{T}-1\right) \lambda, \quad t \in\left[\frac{1}{2} T, T\right],
$$

and introduce the functions $y_{m}:\left[\frac{1}{2} T, T\right] \times \mathbb{R}^{2 n} \rightarrow \mathbb{R}^{n}, m \geq 1$, according to the formula

$$
\begin{aligned}
y_{m}(t, \eta, \lambda):= & \int_{\frac{T}{2}}^{t} f\left(s, y_{m-1}(s, \eta, \lambda)\right) d s-\left(\frac{2 t}{T}-1\right) \int_{\frac{T}{2}}^{T} f\left(s, y_{m-1}(s, \eta, \lambda)\right) d s \\
& +\eta-\left(\frac{2 t}{T}-1\right) \lambda, \quad t \in\left[\frac{1}{2} T, T\right]
\end{aligned}
$$

for all $\eta$ and $\lambda$ from $\mathbb{R}^{n}$.

Let $\mathscr{D}$ be the set appearing in Theorem 7.1. Then the following statement on sequence (7.17) holds. 
Theorem 7.3. Assume that the vector-function $f$ satisfies conditions (3.5), (7.7), and, moreover,

$$
D\left(\frac{T}{8} \delta_{\left[\frac{1}{2} T, T\right], D}(f)+\operatorname{diam} \mathscr{D}\right) \neq \varnothing .
$$

Then, for all fixed $\lambda \in \mathscr{D}-\mathscr{D}$ and $\eta \in D\left(\frac{T}{8} \delta_{\left[\frac{1}{2} T, T\right], D}(f)+\operatorname{diam} \mathscr{D}\right)$ :

(1) For any $m \geq 1, y_{m}(\cdot, \eta, \lambda)$ satisfies the boundary conditions

$$
y_{m}(T, \eta, \lambda)-y_{m}\left(\frac{1}{2} T, \eta, \lambda\right)=-\lambda .
$$

(2) The uniform, in $t \in\left[\frac{1}{2} T, T\right]$, limit

$$
\lim _{m \rightarrow \infty} y_{m}(t, \eta, \lambda)=: y_{\infty}(t, \eta, \lambda)
$$

exists, and moreover,

$$
y_{\infty}(T, \eta, \lambda)-y_{\infty}\left(\frac{1}{2} T, \eta, \lambda\right)=-\lambda .
$$

(3) The function $y_{\infty}(\cdot, \eta, \lambda)$ is the unique solution of the Cauchy problem

$$
\begin{gathered}
y^{\prime}(t)=f(t, y(t))+2 T^{-1} \mathrm{H}(\eta, \lambda), \\
y\left(\frac{1}{2} T\right)=\eta,
\end{gathered}
$$

where

$$
\mathrm{H}(\eta, \lambda):=-\lambda-\int_{\frac{T}{2}}^{T} f\left(\tau, y_{\infty}(\tau, \eta, \lambda)\right) d \tau .
$$

(4) For an arbitrarily small positive $\varepsilon$, one can find a number $m_{\varepsilon} \geq 1$ such that the function

$$
\psi_{m}(\cdot, \eta, \lambda):=y_{m}(\cdot, \eta, \lambda)-y_{\infty}(t, \eta, \lambda)
$$

satisfies the estimate

$$
\left|\psi_{m}(t, \eta, \lambda)\right| \leq \frac{1}{2} \overline{\bar{\alpha}}_{m_{\varepsilon}}(t) K^{m_{\varepsilon}-1}\left(\frac{1}{2} T c_{\varepsilon} K\right)^{m-m_{\varepsilon}+1}\left(1_{n}-\frac{1}{2} T c_{\varepsilon} K\right)^{-1} \delta_{\left[\frac{1}{2} T, T\right], D}(f)
$$

for all $t \in\left[\frac{1}{2} T, T\right]$ and $m \geq m_{\varepsilon}$, where $c_{\varepsilon}$ is given by (5.8).

Remark 7.4. By analogy to Remark 7.2, one can conclude that the validity of estimate (7.25) is ensured for all $m \geq 1$ provided that $\varepsilon \geq \varepsilon_{0}$ with $\varepsilon_{0}$ given by formula (5.11).

Theorems 7.1 and 7.3 suggest that sequences (7.2) and (7.17) can be used to construct the solutions of auxiliary problems (6.1), (6.2) and (6.3), (6.4), and ultimately of the original problem (3.3), (3.4).

Remark 7.5. As will be seen from the proofs (Section 8), Theorems 7.1 and 7.3 could have been formulated so that the inclusion $\lambda \in \mathscr{D}-\mathscr{D}$ would be replaced by $\lambda \in$ $\Lambda_{0}$ (Theorem 7.1) and $-\lambda \in \Lambda_{1}$ (Theorem 7.3), where $\Lambda_{0}$ and $\Lambda_{1}$ are certain sets in 
$\mathbb{R}^{n}$ where the unknown values of $\lambda$ and $-\lambda$ are expected to belong. Assumptions (7.8) and (7.18) would then be replaced by the conditions

$$
\begin{aligned}
& D\left(\frac{T}{8} \delta_{\left[0, \frac{1}{2} T\right], D}(f)+d\left(\Lambda_{0}\right)\right) \neq \varnothing, \\
& D\left(\frac{T}{8} \delta_{\left[\frac{1}{2} T, T\right], D}(f)+d\left(\Lambda_{1}\right)\right) \neq \varnothing,
\end{aligned}
$$

where

$$
d(Q):=\sup \{|z|: z \in Q\}
$$

for any non-empty $Q \subset \mathbb{R}^{n}$

The present choice $\Lambda_{0}=\mathscr{D}-\mathscr{D}, \Lambda_{1}=\mathscr{D}-\mathscr{D}$, with $\mathscr{D} \subset D$, is rather natural because, as follows from the form of the auxiliary boundary conditions (6.2) and (6.4), the looked-for values of $\lambda$ are, in fact, always differences of values of the solutions at some of the points $0, \frac{1}{2} T, T$, and all those values should lie in $D$.

The last two statements are summarised in the following

Theorem 7.6. Assume that the vector-function $f$ satisfies conditions (3.5), (7.7), and, moreover,

$$
D\left(\frac{T}{8} \max \left\{\delta_{\left[0, \frac{1}{2} T\right], D}(f), \delta_{\left[\frac{1}{2} T, T\right], D}(f)\right\}+\operatorname{diam} \mathscr{D}\right) \neq \varnothing,
$$

Then, for any $\xi \in D\left(\frac{T}{8} \delta_{\left[0, \frac{1}{2} T\right], D}(f)+\operatorname{diam} \mathscr{D}\right), \eta \in D\left(\frac{T}{8} \delta_{\left[\frac{1}{2} T, T\right], D}(f)+\operatorname{diam} \mathscr{D}\right)$, and $\lambda \in \mathscr{D}-\mathscr{D}$, the assertions of Theorems 7.1 and 7.3 hold.

\section{Proof of CONVERGENCE THEOREMS}

The proof of Theorems 7.1 and 7.3, which will be given in Section 8.4, uses the fact that, by modifying the problem suitably, the inhomogeneous terms in the boundary conditions (6.2) and (6.4) can be removed so that techniques similar to Proposition 5.1 can be applied.

\subsection{Making the boundary conditions periodic}

Let $\mathscr{D}$ be the set appearing in the formulation of Theorem 7.1. For any fixed $\lambda \in \mathscr{D}-\mathscr{D}$, consider the boundary value problems

$$
\begin{gathered}
\tilde{x}^{\prime}(t)=f\left(t, \tilde{x}(t)+\frac{2 t}{T} \lambda\right)-\frac{2}{T} \lambda, \quad t \in\left[0, \frac{1}{2} T\right], \\
\tilde{x}\left(\frac{T}{2}\right)-\tilde{x}(0)=0
\end{gathered}
$$

and

$$
\begin{gathered}
\tilde{y}^{\prime}(t)=f\left(t, \tilde{y}(t)+\left(1-\frac{2 t}{T}\right) \lambda\right)+\frac{2}{T} \lambda, \quad t \in\left[\frac{1}{2} T, T\right], \\
\tilde{y}(T)-\tilde{y}\left(\frac{T}{2}\right)=0 .
\end{gathered}
$$

Then, by direct computation, one easily finds that 
Lemma 8.1. The following assertions are true for an arbitrary $\lambda \in \mathbb{R}^{n}$ :

(1) A function $\tilde{x}:\left[0, \frac{1}{2} T\right] \rightarrow \mathbb{R}^{n}$ satisfies (8.1), (8.2) if and only if the function

$$
x(t):=\tilde{x}(t)+\frac{2 t}{T} \lambda, \quad t \in\left[0, \frac{1}{2} T\right],
$$

is a solution of problem (6.1), (6.2).

(2) A function $\tilde{y}:\left[\frac{1}{2} T, T\right] \rightarrow \mathbb{R}^{n}$ satisfies (8.3), (8.4) if and only if the function

$$
y(t):=\tilde{y}(t)+\left(1-\frac{2 t}{T}\right) \lambda, \quad t \in\left[\frac{1}{2} T, T\right],
$$

is a solution of problem (6.3), (6.4).

Keeping this equivalence in mind, one can deal with problems (6.1), (6.2) and (6.3), (6.4) by using Proposition 5.1, for which purpose the corresponding iteration process should be constructed. Indeed, for any fixed $\lambda \in \mathbb{R}^{n}$ and $(\xi, \eta) \in D^{2}$, let us put

$$
\begin{aligned}
\tilde{x}_{m}(t, \xi, \lambda):= & \xi+\int_{0}^{t} f\left(s, \tilde{x}_{m-1}(s, \xi, \lambda)+\frac{2 s}{T} \lambda\right) d s \\
& -\frac{2 t}{T} \int_{0}^{\frac{T}{2}} f\left(s, \tilde{x}_{m-1}(s, \xi, \lambda)+\frac{2 s}{T} \lambda\right) d s, \quad t \in\left[0, \frac{1}{2} T\right]
\end{aligned}
$$

and

$$
\begin{aligned}
\tilde{y}_{m}(t, \eta, \lambda):= & \eta+\int_{\frac{T}{2}}^{t} f\left(s, \tilde{y}_{m-1}(s, \eta, \lambda)+\left(1-\frac{2 s}{T}\right) \lambda\right) d s \\
& -\left(\frac{2 t}{T}-1\right) \int_{\frac{T}{2}}^{T} f\left(s, \tilde{y}_{m-1}(s, \eta, \lambda)+\left(1-\frac{2 s}{T}\right) \lambda\right) d s, \quad t \in\left[\frac{1}{2} T, T\right],
\end{aligned}
$$

for all $m=1,2, \ldots$, where

$$
\begin{array}{ll}
\tilde{x}_{0}(t, \xi, \lambda):=\xi, & t \in\left[0, \frac{1}{2} T\right], \\
\tilde{y}_{0}(t, \eta, \lambda):=\eta, & t \in\left[\frac{1}{2} T, T\right] .
\end{array}
$$

Similarly to Proposition 5.1, we see that functions (8.7) and (8.8) have the properties

$$
\begin{aligned}
\tilde{x}_{m}(0, \xi, \lambda) & =\tilde{x}_{m}\left(\frac{T}{2}, \xi, \lambda\right), \\
\tilde{y}_{m}\left(\frac{T}{2}, \eta, \lambda\right) & =\tilde{y}_{m}(T, \eta, \lambda)
\end{aligned}
$$

for all $\xi, \eta$, and $m$. 


\subsection{Estimates of non-linearities}

For any fixed $\lambda \in \mathscr{D}-\mathscr{D}$, put

$$
D_{\lambda, i}:=\left\{z \in \mathbb{R}^{n}: z+(-1)^{i} \theta \lambda \in D \text { for all } \theta \in[0,1]\right\}
$$

for $i \in\{0,1\}$.

Lemma 8.2. Let $\lambda \in \mathscr{D}-\mathscr{D}$ be arbitrary. Then:

(1) $\left\{\xi+2 t T^{-1} \lambda: t \in\left[0, \frac{1}{2} T\right]\right\} \subset D$ if and only if $\xi \in D_{\lambda, 0}$.

(2) $\left\{\eta+\left(1-2 t T^{-1}\right) \lambda: t \in\left[\frac{1}{2} T, T\right]\right\} \subset D$ if and only if $\eta \in D_{\lambda, 1}$.

Proof. Indeed, if $\xi \in D_{\lambda, 0}$, then, according to (8.11), $z+\theta \lambda \in D$ for all $\theta \in[0,1]$, and therefore $\xi+2 T^{-1} \lambda t \in D$ for any $t \in\left[0, \frac{1}{2} T\right]$. Similarly, given an $\eta \in D_{\lambda, 1}$, we have $\eta-\theta \lambda \in D$ for all $\theta \in[0,1]$ and, hence, $\eta+\left(1-2 t T^{-1}\right) \lambda \in D$ for $t \in$ $\left[\frac{1}{2} T, T\right]$.

When estimating the integral terms in (8.7) and (8.8), the following result will be used.

Lemma 8.3 ([19]). Let $u:[0, T] \rightarrow \mathbb{R}^{n}$ be continuous. The following estimates are true:

$$
\int_{0}^{t}\left(u(s)-\frac{2}{T} \int_{0}^{\frac{T}{2}} u(\sigma) d \sigma\right) d s \leq \frac{1}{2} \bar{\alpha}_{1}(t) \max _{s \in[0, t], \sigma \in\left[t, \frac{1}{2} T\right]}|u(s)-u(\sigma)|
$$

for $t \in\left[0, \frac{1}{2} T\right]$ and

$$
\int_{\frac{T}{2}}^{t}\left(u(s)-\frac{2}{T} \int_{\frac{T}{2}}^{T} u(\sigma) d \sigma\right) d s \leq \frac{1}{2} \overline{\bar{\alpha}}_{1}(t) \max _{s \in\left[\frac{1}{2} T, t\right], \sigma \in[t, T]}|u(s)-u(\sigma)|
$$

for $t \in\left[\frac{1}{2} T, T\right]$.

Here, as before, the inequalities between vectors and the operation "max" for vector functions are understood in the component-wise sense.

Proof of Lemma 8.3. The assertion is obtained along the lines of proof of [19, Lemma 3] after rescaling the original interval to $\left[0, \frac{1}{2} T\right]$ and $\left[\frac{1}{2} T, T\right]$ respectively. The main points of the proof being the same, the details are omitted.

Given arbitrary $i \in\{0,1\}$ and $y \in C\left(\left[\frac{1}{2} i T, \frac{1}{2}(i+1) T\right], \mathbb{R}^{n}\right)$, put

$$
\left(P_{i} v\right)(t):=\int_{\frac{i}{2} T}^{t} v(s) d s-\left(\frac{2 t}{T}-i\right) \int_{\frac{i}{2} T}^{\frac{i+1}{2} T} v(s) d s
$$

for all $t \in\left[\frac{1}{2} i T, \frac{1}{2}(i+1) T\right]$. It is clear that the linear mapping $P_{i}$ transforms the space $C\left(\left[\frac{1}{2} i T, \frac{1}{2}(i+1) T\right], \mathbb{R}^{n}\right)$ to itself. 
Lemma 8.4. The estimate

$$
\left|\left(P_{0} v\right)(t)\right| \leq\left(1+i-\frac{2 t}{T}\right) \int_{\frac{i}{2} T}^{t}|v(s)| d s+\left(\frac{2 t}{T}-i\right) \int_{t}^{\frac{i+1}{2} T}|v(s)| d s
$$

holds for any $i \in\{0,1\}, v \in C\left(\left[\frac{1}{2} i T, \frac{1}{2}(i+1) T\right], \mathbb{R}^{n}\right)$, and $t \in\left[\frac{1}{2} i T, \frac{1}{2}(i+1) T\right]$.

Proof. Estimate (8.15) is established by direct computation using (8.14).

Now define the functions $X_{\lambda}:\left[0, \frac{1}{2} T\right] \times D \rightarrow \mathbb{R}^{n}$ and $Y_{\lambda}:\left[\frac{1}{2} T, T\right] \times D \rightarrow \mathbb{R}^{n}$ by setting

$$
X_{\lambda}(t, \xi):=f\left(t, \xi+\frac{2 t}{T} \lambda\right), \quad(t, \xi) \in\left[0, \frac{1}{2} T\right] \times D,
$$

and

$$
Y_{\lambda}(t, \eta):=f\left(t, \eta+\left(1-\frac{2 t}{T}\right) \lambda\right), \quad(t, \eta) \in\left[\frac{1}{2} T, T\right] \times D .
$$

Lemma 8.5. Let $\lambda \in \mathscr{D}-\mathscr{D}$, and $u:\left[0, \frac{1}{2} T\right] \rightarrow \mathbb{R}^{n}, v:\left[\frac{1}{2} T, T\right] \rightarrow \mathbb{R}^{n}$ be arbitrary functions such that $\left\{u(t): t \in\left[0, \frac{1}{2} T\right]\right\} \subset D_{\lambda, 0}$ and $\left\{v(t): t \in\left[\frac{1}{2} T, T\right]\right\} \subset D_{\lambda, 1}$. Then:

(1) For $t \in\left[0, \frac{1}{2} T\right]$,

$$
\begin{aligned}
\left|P_{0} X_{\lambda}(\cdot, u(\cdot))\right|(t) & \leq \frac{1}{2} \bar{\alpha}_{1}(t) \delta_{\left[0, \frac{1}{2} T\right], D}(f) \\
& \leq \frac{T}{8} \delta_{\left[0, \frac{1}{2} T\right], D}(f)
\end{aligned}
$$

(2) For $t \in\left[\frac{1}{2} T, T\right]$,

$$
\begin{aligned}
\left|P_{1} Y_{\lambda}(\cdot, v(\cdot))\right|(t) & \leq \frac{1}{2} \overline{\bar{\alpha}}_{1}(t) \delta_{\left[\frac{1}{2} T, T\right], D}(f) \\
& \leq \frac{T}{8} \delta_{\left[\frac{1}{2} T, T\right], D}(f) .
\end{aligned}
$$

Recall that we use notation (4.2).

Proof. Let us fix a $\lambda \in \mathscr{D}-\mathscr{D}$. Let us prove, e.g., inequality (8.19). Take an $\eta \in D_{\lambda, 1}$. Applying inequality (8.13) of Lemma 8.3 with $u=Y_{\lambda}(\cdot, \eta)$, we obtain the estimate

$$
\left|\left(P_{1} Y_{\lambda}(\cdot, v(\cdot))\right)(t)\right| \leq \frac{1}{2} \overline{\bar{\alpha}}_{1}(t) \max _{s \in\left[\frac{1}{2} T, t\right], \sigma \in[t, T]}\left|Y_{\lambda}(s, v(s))-Y_{\lambda}(\sigma, v(\sigma))\right|
$$

valid for $t \in\left[\frac{1}{2} T, T\right]$. Since $v$ has values in $D_{\lambda, 1}$, Lemma 8.2 ensures that $v(t)+$ $2 t T^{-1} \lambda \in D$ for all $t \in\left[0, \frac{1}{2} T\right]$ and, therefore, considering (8.17), we see that the 
Lipschitz condition (3.5) assumed for $f$ can be applied in (8.20). Then (8.17) and (8.20) yield the estimate

$$
\left|\left(P_{1} Y_{\lambda}(\cdot, v(\cdot))\right)(t)\right| \leq \frac{1}{2} \overline{\bar{\alpha}}_{1}(t) \delta_{\left[\frac{1}{2} T, T\right], D}(f), \quad t \in\left[\frac{1}{2} T, T\right] .
$$

It now remains to observe that the maximal value of the function $\overline{\bar{\alpha}}_{1}$ given by equality (7.6) is attained at $\frac{3}{4} T$ and

$$
\max _{t \in\left[\frac{1}{2} T, T\right]} \overline{\bar{\alpha}}_{1}(t)=\frac{T}{4},
$$

which leads us immediately to (8.13).

Inequality (8.18) is proved similarly by estimating the value of $P_{0}$ on the function $X_{\lambda}(\cdot, u(\cdot))$ with $u\left(\left[0, \frac{1}{2} T\right]\right) \subset D_{\lambda, 0}$ and using the equality

$$
\max _{t \in\left[0, \frac{1}{2} T\right]} \bar{\alpha}_{1}(t)=\frac{T}{4}
$$

which is obtained directly from formula (7.5) using the fact that $\max _{t \in\left[0, \frac{1}{2} T\right]} \bar{\alpha}_{1}(t)=$ $\bar{\alpha}_{1}\left(\frac{1}{2}\right)$. Indeed, similarly to (8.21), one shows that

$$
\left|\left(P_{0} X_{\lambda}(\cdot, u(\cdot))\right)(t)\right| \leq \frac{1}{2} \bar{\alpha}_{1}(t) \delta_{\left[0, \frac{1}{2} T\right], D}(f), \quad t \in\left[0, \frac{1}{2} T\right] .
$$

The required estimate (8.18) is then a direct consequence of relations (8.23) and (8.24). Inequality (8.18) is established in a similar way.

\subsection{Functions $\bar{\alpha}_{m}$ and $\overline{\bar{\alpha}}_{m}, m=0,1, \ldots$}

We need the following estimates for the sequences of functions (7.3) and (7.4).

Lemma 8.6. Given any $\varepsilon \in(0,+\infty)$, one can specify an integer $m_{\varepsilon} \geq 1$ such that the estimates

$$
\bar{\alpha}_{m+1}(t) \leq \frac{1}{2} T\left(\varrho_{*}+\varepsilon\right) \bar{\alpha}_{m}(t), \quad t \in\left[0, \frac{1}{2} T\right]
$$

and

$$
\overline{\bar{\alpha}}_{m+1}(t) \leq \frac{1}{2} T\left(\varrho_{*}+\varepsilon\right) \overline{\bar{\alpha}}_{m}(t), \quad t \in\left[\frac{1}{2} T, T\right],
$$

hold for all $m \geq m_{\mathcal{E}}$, where $\varrho_{*}$ is defined by (4.3).

Proof. Estimate (8.25) (resp., (8.26)) is obtained directly from Lemma 5.3 by putting $t_{0}:=0\left(\right.$ resp., $\left.t_{0}:=\frac{1}{2} T\right)$ and $p:=\frac{1}{2} T$ in (5.9).

Remark 8.7. It follows immediately from (8.25) and (8.26) that

$$
\bar{\alpha}_{m+1}(t) \leq\left(\frac{T}{2}\right)^{m-m_{\varepsilon}+1}\left(\varrho_{*}+\varepsilon\right)^{m-m_{\varepsilon}+1} \bar{\alpha}_{m_{\varepsilon}}(t), \quad t \in\left[0, \frac{1}{2} T\right],
$$


and

$$
\overline{\bar{\alpha}}_{m+1}(t) \leq\left(\frac{T}{2}\right)^{m-m_{\varepsilon}+1}\left(\varrho_{*}+\varepsilon\right)^{m-m_{\varepsilon}+1} \overline{\bar{\alpha}}_{m_{\varepsilon}}(t), \quad t \in\left[\frac{1}{2} T, T\right],
$$

for all $m \geq m_{\varepsilon}$, where $m_{\varepsilon}$ is the integer appearing in Lemma 8.6. Inequalities (8.27) and (8.28) improve the corresponding estimate from the work [20].

\subsection{Proofs of Theorems 7.1 and 7.3}

We shall use an approach similar to that described in Proposition 5.1 adopting it appropriately to problems (6.1), (6.2) and (6.3), (6.4).

Proof of Theorem 7.1. Let $\lambda \in \mathscr{D}-\mathscr{D}$ and

$$
\xi \in D\left(\frac{T}{8} \delta_{\left[0, \frac{1}{2} T\right], D}(f)+\operatorname{diam} \mathscr{D}\right)
$$

be fixed. It is not difficult to verify by computation that $\tilde{x}_{m}(\cdot, \xi, \lambda), m \geq 0$, coincides with sequence (5.1) for $t_{0}=0, p=\frac{1}{2} T$, and $g=X_{\lambda}-2 T^{-1} \lambda$, where the function $X_{\lambda}:\left[0, \frac{1}{2} T\right] \times D \rightarrow \mathbb{R}^{n}$ is defined according to (8.16).

Let us show that

$$
\left\{\tilde{x}_{m}(t, \xi, \lambda): t \in\left[0, \frac{1}{2} T\right]\right\} \subset D_{\lambda, 0}
$$

for all $m \geq 0$. For $m=0$, the validity of inclusion (8.30) is guaranteed directly by (8.9) and (8.29). Indeed, for all $\theta \in[0,1]$ and $z$ such that

$$
|\xi-z| \leq \frac{T}{8} \delta_{\left[0, \frac{1}{2} T\right], D}(f)
$$

the estimates

$$
\begin{aligned}
|\xi+\theta \lambda-z| & \leq|\xi-z|+|\lambda| \\
& \leq|\xi-z|+\operatorname{diam} \mathscr{D} \\
& \leq \frac{T}{8} \delta_{\left[0, \frac{1}{2} T\right], D}(f)+\operatorname{diam} \mathscr{D}
\end{aligned}
$$

hold. In (8.31), we have used the assumption that $\lambda$ belongs to $\mathscr{D}-\mathscr{D}$. By virtue of (8.29), inequality (8.31) guarantees that $z+\theta \lambda \in D$, i. e., $\xi \in D_{\lambda, 0}$ and (8.30) holds with $m=0$.

Assume that (8.30) holds for a certain value of $m$ and show that the inclusion

$$
\left\{\tilde{x}_{m+1}(t, \xi, \lambda): t \in\left[0, \frac{1}{2} T\right]\right\} \subset D_{\lambda, 0}
$$

holds as well.

Indeed, considering definition (8.7) and recalling notation (8.14), we conclude that, for all $m$, the identity

$$
\tilde{x}_{m+1}(\cdot, \xi, \lambda)-\xi=P_{0} X_{\lambda}\left(\cdot, \tilde{x}_{m}(\cdot, \xi, \lambda)\right)
$$


holds. Here, we have used the fact that $P_{0} c=0$ for every $c \in \mathbb{R}^{n}$. Therefore, taking assumption (8.30) and inequality (8.18) of Lemma 8.5 into account, we obtain the estimates

$$
\left|\tilde{x}_{m+1}(t, \xi, \lambda)-\xi\right| \leq \frac{T}{8} \delta_{\left[0, \frac{1}{2} T\right], D}(f)
$$

valid for all $t \in\left[0, \frac{1}{2} T\right]$.

Therefore,

$$
\begin{aligned}
\left|\tilde{x}_{m+1}(t, \xi, \lambda)+\theta \lambda-\xi\right| & \leq \frac{T}{8} \delta_{\left[0, \frac{1}{2} T\right], D}(f)+|\lambda| \\
& \leq \frac{T}{8} \delta_{\left[0, \frac{1}{2} T\right], D}(f)+\operatorname{diam} \mathscr{D}
\end{aligned}
$$

for all $\theta \in[0,1]$ and $t \in\left[0, \frac{1}{2} T\right]$. However, in view of notation (4.1), estimate (8.34) implies that

$$
\left\{\tilde{x}_{m+1}(t, \xi, \lambda)+\theta \lambda: \theta \in[0,1], t \in\left[0, \frac{1}{2} T\right]\right\} \subset D,
$$

which means that (8.32) holds. Thus, inclusion (8.30) is true for all $m \geq 0$.

Now, following [8], consider the differences

$$
\bar{d}_{m}(t, \xi, \lambda):=\tilde{x}_{m+1}(t, \xi, \lambda)-\tilde{x}_{m}(t, \xi, \lambda), \quad m=0,1, \ldots
$$

By virtue of (8.33) and Lemma 8.5,

$$
\left|\bar{d}_{0}(t, \xi, \lambda)\right| \leq \frac{1}{2} \bar{\alpha}_{1}(t) \delta_{\left[0, \frac{1}{2} T\right], D}(f), \quad t \in\left[0, \frac{1}{2} T\right] .
$$

In view of (8.33),

$$
\bar{d}_{m}(\cdot, \xi, \lambda)=P_{0}\left(X_{\lambda}\left(\cdot, \tilde{x}_{m}(\cdot, \xi, \lambda)\right)-X_{\lambda}\left(\cdot, \tilde{x}_{m-1}(\cdot, \xi, \lambda)\right)\right)
$$

for any $m$. Since (8.30) holds for all $m$, it follows that, for any $t \in\left[0, \frac{1}{2} T\right]$, the function $X_{\lambda}(t, \cdot)$ satisfies the Lipschitz condition with the same matrix $K$ as $f(t, \cdot)$ does. Therefore, relations (8.37), (8.36), Lemma 8.4, and notation (7.3) yield

$$
\begin{aligned}
\left|\bar{d}_{1}(t, \xi, \lambda)\right| & \leq\left(1-\frac{2 t}{T}\right) K \int_{0}^{t}\left|\bar{d}_{0}(s, \xi, \lambda)\right| d s+\frac{2 t}{T} K \int_{t}^{\frac{1}{2} T}\left|\bar{d}_{0}(s, \xi, \lambda)\right| d s \\
& \leq \frac{1}{2}\left(\left(1-\frac{2 t}{T}\right) K \int_{0}^{t} \bar{\alpha}_{1}(s) d s+\frac{2 t}{T} K \int_{t}^{\frac{1}{2} T} \bar{\alpha}_{1}(s) d s\right) \delta_{\left[0, \frac{1}{2} T\right], D}(f) \\
& =\frac{1}{2} \bar{\alpha}_{2}(t) K \delta_{\left[0, \frac{1}{2} T\right], D}(f)
\end{aligned}
$$


for $t \in\left[0, \frac{1}{2} T\right]$. On the other hand, considering (8.37) and using the Lipschitz condition, we obtain the inequality

$$
\begin{aligned}
\left|\bar{d}_{m}(t, \xi, \lambda)\right| \leq\left(1-\frac{2 t}{T}\right) & K \int_{0}^{t}\left|\bar{d}_{m-1}(s, \xi, \lambda)\right| d s \\
& +\frac{2 t}{T} K \int_{t}^{\frac{1}{2} T}\left|\bar{d}_{m-1}(s, \xi, \lambda)\right| d s, \quad t \in\left[0, \frac{1}{2} T\right],
\end{aligned}
$$

for $m \geq 1$. Combining (8.36) and (8.39), recalling notation (7.3), and arguing by induction, we arrive to the estimate

$$
\left|\bar{d}_{m}(t, \xi, \lambda)\right| \leq \frac{1}{2} K^{m} \bar{\alpha}_{m+1}(t) \delta_{\left[0, \frac{1}{2} T\right], D}(f)
$$

valid for all $t \in\left[0, \frac{1}{2} T\right]$. It follows from assumption (7.7) that, for a sufficiently small positive number $\varepsilon$, the inequality

$$
r(K)<\frac{2}{T\left(\varrho_{*}+\varepsilon\right)}
$$

holds. Let us fix such an $\varepsilon$ and let $m \geq m_{\varepsilon}$, where $m_{\varepsilon}$ is the integer appearing in Lemma 8.6. Then (8.27) and (8.40) yield

$$
\left|\bar{d}_{m+1}(t, \xi, \lambda)\right| \leq \frac{T^{m-m_{\varepsilon}+2}}{2^{m-m_{\varepsilon}+3}} K^{m+1}\left(\varrho_{*}+\varepsilon\right)^{m-m_{\varepsilon}+2} \bar{\alpha}_{m_{\varepsilon}}(t) \delta_{\left[0, \frac{1}{2} T\right], D}(f)
$$

for $t \in\left[0, \frac{1}{2} T\right]$. Now note that, due to (8.41), we have

$$
\lim _{m \rightarrow+\infty} 2^{-m} T^{m}\left(\varrho_{*}+\varepsilon\right)^{m} K^{m}=0 .
$$

Then, arguing similarly to the proof of [8, Theorem 1], we conclude that (8.42) and (8.43) imply the convergence of the sequence $\tilde{x}_{m}(\cdot, \xi, \lambda), m \geq 0$. Since (7.2) and (8.7) imply the identity

$$
x_{m}(t, \xi, \lambda)=\tilde{x}_{m}(t, \xi, \lambda)+\frac{2 t}{T} \lambda, \quad t \in\left[0, \frac{1}{2} T\right],
$$

the latter fact is equivalent to the convergence of $x_{m}(\cdot, \xi, \lambda), m \geq 0$. The rest of the proof almost literally follows the lines of [8].

Proof of Theorem 7.3. It follows along the lines of proof of Theorem 7.1. One finds that $\tilde{y}_{m}(\cdot, \eta, \lambda), m \geq 0$, is nothing but (5.1) with $t_{0}=\frac{1}{2} T, p=\frac{1}{2} T$, and $g=$ $Y_{\lambda}+2 T^{-1} \lambda$, where $Y_{\lambda}:\left[\frac{1}{2} T, T\right] \times D \rightarrow \mathbb{R}^{n}$ is given by (8.17). The required assertion is established by analogy, instead of (8.44), using the identity

$$
y_{m}(t, \eta, \lambda)=\tilde{y}_{m}(t, \eta, \lambda)-\frac{2 t}{T} \lambda, \quad t \in\left[\frac{1}{2} T, T\right],
$$

which follows, for any $\eta \in D\left(\frac{T}{8} \delta_{\left[\frac{1}{2} T, T\right], D}(f)+\operatorname{diam} \mathscr{D}\right)$, directly from relations (7.17) and (8.8). 


\section{Determining EQUATIONS}

Theorems 7.1 and 7.3 guarantee that, under the conditions assumed, the functions $x_{\infty}(\cdot, \xi, \lambda):\left[0, \frac{1}{2} T\right] \rightarrow \mathbb{R}^{n}$ and $y_{\infty}(\cdot, \eta, \lambda):\left[\frac{1}{2} T, T\right] \rightarrow \mathbb{R}^{n}$ are well-defined for the appropriate values of $(\xi, \eta, \lambda)$. Therefore, one can introduce the function $u_{\infty}(\cdot, \xi, \eta, \lambda)$ : $[0, T] \rightarrow \mathbb{R}^{n}$ by putting

$$
\begin{aligned}
u_{\infty}(t, \xi, \eta, \lambda):= & \chi_{T}(t) x_{\infty}(t, \xi, \lambda) \\
& +\left(1-\chi_{T}(t)\right)\left(y_{\infty}(t, \eta, \lambda)-y_{\infty}\left(\frac{1}{2} T, \eta, \lambda\right)+x_{\infty}\left(\frac{1}{2} T, \xi, \lambda\right)\right)
\end{aligned}
$$

for all $t \in[0, T]$ and $\xi \in D\left(\frac{T}{8} \delta_{\left[0, \frac{1}{2} T\right], D}(f)+\operatorname{diam} \mathscr{D}\right), \eta \in D\left(\frac{T}{8} \delta_{\left[\frac{1}{2} T, T\right], D}(f)+\right.$ $\operatorname{diam} \mathscr{D})$, and $\lambda \in \mathscr{D}-\mathscr{D}$.

Theorem 9.1. Assume that $f$ satisfies the Lipschitz condition (3.5) with a matrix $K$ such that (7.7) holds. Furthermore, let

$$
D\left(\frac{T}{8} \delta_{[0, T], D}(f)+\operatorname{diam} \mathscr{D}\right) \neq \varnothing .
$$

Then the following assertions hold:

(1) The function $u_{\infty}(\cdot, \xi, \eta, \lambda):[0, T] \rightarrow \mathbb{R}^{n}$ defined by (9.1) is a solution of the boundary value problem (3.3), (3.4) if and only if the triplet $(\xi, \eta, \lambda)$ satisfies the system of $3 n$ equations

$$
\begin{gathered}
\Xi(\xi, \lambda)=0, \\
\mathrm{H}(\eta, \lambda)=0, \\
x_{\infty}\left(\frac{1}{2} T, \xi, \lambda\right)=\eta .
\end{gathered}
$$

(2) For every solution $u(\cdot)$ of problem (3.3), (3.4) with $u(0)$ and $u\left(\frac{1}{2} T\right)$ lying in $D\left(\frac{T}{8} \delta_{[0, T], D}(f)+\operatorname{diam} \mathscr{D}\right)$, there exists a triplet $\left(\xi_{0}, \eta_{0}, \lambda_{0}\right)$ such that $u(\cdot)=u_{\infty}\left(\cdot, \xi_{0}, \eta_{0}, \lambda_{0}\right)$.

Proof. It follows immediately from (4.1) that $D\left(\beta_{0}\right) \subseteq D\left(\beta_{1}\right)$ whenever $\beta_{0} \leq \beta_{1}$. Therefore, using notation (4.2) and the obvious inequality

$$
\max \left\{\delta_{\left[0, \frac{1}{2} T\right], D}(f), \delta_{\left[\frac{1}{2} T, T\right], D}(f)\right\} \leq \delta_{[0, T], D}(f),
$$

we find that that assumption (9.2) ensures the validity of relations (7.8) and (8.30) and, therefore, Theorems 7.1 and 7.3 can be applied. It then remains to use Proposition 6.1 and argue by analogy to [18, Theorem 5.2].

Equations (9.3) are referred to as determining equations [5,11].

\section{A REMARK ON THE CONSTRUCTIVE APPLICATION OF THE SCHEME}

Although Theorem 9.1 theoretically solves the question on the construction of a solution of the periodic problem (3.3), (3.4), its application faces with difficulties due to the fact that the explicit form of the functions $\Xi: D \times \mathbb{R}^{n} \rightarrow \mathbb{R}^{n}$ and $\mathrm{H}: D \times \mathbb{R}^{n} \rightarrow$ 
$\mathbb{R}^{n}$ appearing in (9.3) is usually unknown. This complication can be overcome by using the functions

$$
\Xi_{m}(\xi, \lambda):=\lambda-\int_{0}^{\frac{T}{2}} f\left(\tau, x_{m}(\tau, \xi, \lambda)\right) d \tau
$$

and

$$
\mathrm{H}_{m}(\eta, \lambda):=-\lambda-\int_{\frac{T}{2}}^{T} f\left(\tau, y_{m}(\tau, \eta, \lambda)\right) d \tau
$$

for a fixed $m$, which will lead one to the so-called approximate determining equations. More precisely, similarly to [15], one can show that, under certain natural assumptions, the exact determining system (9.3) can be replaced by its approximate analogue

$$
\begin{gathered}
\Xi_{m}(\xi, \lambda)=0, \\
\mathrm{H}_{m}(\eta, \lambda)=0, \\
x_{m}\left(\frac{1}{2} T, \xi, \lambda\right)=\eta .
\end{gathered}
$$

It is important to note that, unlike system (9.3), the $m$ th approximate determining system (10.3) contains only terms involving the functions $x_{m}$ and $y_{m}$ and, thus, known explicitly. The topic concerning the use of the approximate determining equations in solvability analysis falls out of the scope of this paper and will be developed elsewhere.

\section{ACKNOWLEDGEMENT}

Research supported in part by RVO: 67985840 (A. Rontó). This research was carried out as part of the TAMOP-4.2.1.B-10/2/KONV-2010-0001 project with support by the European Union, co-financed by the European Social Fund (M. Rontó).

\section{REFERENCES}

[1] V. Berinde, "Existence and approximation of solutions of some first order iterative differential equations," Miskolc Math. Notes, vol. 11, no. 1, pp. 13-26, 2010.

[2] L. Cesari, Asymptotic behavior and stability problems in ordinary differential equations, ser. Second edition. Ergebnisse der Mathematik und ihrer Grenzgebiete, N. F., Bd. 16. New York: Academic Press Inc., 1963.

[3] M. Farkas, Periodic motions, ser. Applied Mathematical Sciences. New York: Springer-Verlag, 1994, vol. 104.

[4] M. Fečkan, "Minimal periods of periodic solutions," Miskolc Math. Notes, vol. 7, no. 2, pp. 121139 (2007), 2006.

[5] R. E. Gaines and J. L. Mawhin, Coincidence degree, and nonlinear differential equations, ser. Lecture Notes in Mathematics, Vol. 568. Berlin: Springer-Verlag, 1977.

[6] J. K. Hale, Oscillations in nonlinear systems. Inc., New York: McGraw-Hill Book Co., 1963. 
[7] I. Kiguradze and S. Mukhigulashvili, "On periodic solutions of two-dimensional nonautonomous differential systems,” Nonlinear Anal., vol. 60, no. 2, pp. 241-256, 2005. [Online]. Available: http://dx.doi.org/10.1016/j.na.2004.07.053

[8] A. Ronto and M. Rontó, "A note on the numerical-analytic method for nonlinear two-point boundary-value problems," Nonlinear Oscil., vol. 4, no. 1, pp. 112-128, 2001.

[9] A. Rontó and M. Rontó, "On some symmetric properties of periodic solutions," Nonlinear Oscil. (N. Y.), vol. 6, no. 1, pp. 82-107, 2003. [Online]. Available: http://dx.doi.org/10.1023/A: 1024827821289

[10] A. Rontó and M. Rontó, "On the $(\tau, E)$ property of periodic solutions," in Colloquium on Differential and Difference Equations, CDDE 2002 (Brno), ser. Folia Fac. Sci. Natur. Univ. Masaryk. Brun. Math. Brno: Masaryk Univ., 2003, vol. 13, pp. 247-267.

[11] A. Rontó and M. Rontó, "Successive approximation techniques in non-linear boundary value problems for ordinary differential equations," in Handbook of differential equations: ordinary differential equations. Vol. IV, ser. Handb. Differ. Equ. Elsevier/North-Holland, Amsterdam, 2008, pp. 441-592.

[12] A. Rontó and M. Rontó, "On a Cauchy-Nicoletti type three-point boundary value problem for linear differential equations with argument deviations," Miskolc Math. Notes, vol. 10, no. 2, pp. 173-205, 2009.

[13] A. Rontó and M. Rontó, "Successive approximation method for some linear boundary value problems for differential equations with a special type of argument deviation," Miskolc Math. Notes, vol. 10, no. 1, pp. 69-95, 2009.

[14] A. Rontó and M. Rontó, "On nonseparated three-point boundary value problems for linear functional differential equations," Abstr. Appl. Anal., pp. Art. ID 326052, 22, 2011. [Online]. Available: http://dx.doi.org/10.1155/2011/326052

[15] A. Rontó and M. Rontó, "Existence results for three-point boundary value problems for systems of linear functional differential equations," Carpathian J. Math., vol. 28, no. 1, pp. 163-182, 2012.

[16] A. N. Ronto, M. Rontó, A. M. Samoilenko, and S. I. Trofimchuk, "On periodic solutions of autonomous difference equations," Georgian Math. J., vol. 8, no. 1, pp. 135-164, 2001.

[17] A. N. Ronto, M. Ronto, and N. M. Shchobak, "On the parametrization of three-point nonlinear boundary value problems," Nonlinear Oscil., vol. 7, no. 3, pp. 384-402, 2004.

[18] M. Rontó and K. Marinets, "On the parametrization of boundary value problems with two-point nonlinear boundary conditions," Nelīnĭñ̄ Koliv., vol. 14, no. 3, pp. 359-391, 2011.

[19] M. Rontó and J. Mészáros, "Some remarks on the convergence of the numerical-analytical method of successive approximations," Ukrainian Math. J., vol. 48, no. 1, pp. 101-107, 1996. [Online]. Available: http://dx.doi.org/10.1007/BF02390987

[20] M. Rontó and A. M. Samoilenko, Numerical-analytic methods in the theory of boundary-value problems. River Edge, NJ: World Scientific Publishing Co. Inc., 2000, with a preface by Yu. A. Mitropolsky and an appendix by the authors and S. I. Trofimchuk. [Online]. Available: http://dx.doi.org/10.1142/9789812813602

[21] M. Rontó and N. Shchobak, "On the numerical-analytic investigation of parametrized problems with nonlinear boundary conditions," Nonlinear Oscil. (N. Y.), vol. 6, no. 4, pp. 469-496, 2003. [Online]. Available: http://dx.doi.org/10.1023/B:NONO.0000028586.11256.d7

[22] M. Rontó and N. Shchobak, "On parametrization for a non-linear boundary value problem with separated conditions," in The 8th Colloquium on the Qualitative Theory of Differential Equations, ser. Proc. Colloq. Qual. Theory Differ. Equ. Electron. J. Qual. Theory Differ. Equ., Szeged, 2008, vol. 8, pp. No. 18, 16.

[23] N. I. Ronto, A. M. Samoilenko, and S. I. Trofimchuk, "The theory of the numerical-analytic method: achievements and new directions of development. VII," Ukrainian Math. J., vol. 51, no. 9, pp. 1399-1418 (2000), 1999. [Online]. Available: http://dx.doi.org/10.1007/BF02593006 
[24] A. M. Samoilenko, "A numerical-analytic method for investigation of periodic systems of ordinary differential equations. I," Ukrain. Mat. Zh., vol. 17, no. 4, pp. 82-93, 1965.

[25] A. M. Samoilenko, "A numerical-analytic method for investigation of periodic systems of ordinary differential equations. II," Ukrain. Mat. Zh., vol. 18, no. 2, pp. 50-59, 1966.

[26] A. M. Samoilenko, "On a sequence of polynomials and the radius of convergence of its Abel-Poisson sum," Ukrainian Math. J., vol. 55, no. 7, pp. 1119-1130, 2003. [Online]. Available: http://dx.doi.org/10.1023/B:UKMA.0000010610.69570.13

[27] A. M. Samoilenko and N. I. Ronto, Numerical-analytic methods of investigating periodic solutions. Moscow: "Mir", 1979, translated from the Russian by Vladimir Shokurov [Vladimir Šokurov], With a foreword by Yu. A. Mitropolskii.

[28] A. M. Samoilenko and N. I. Ronto, Numerical-analytic methods of investigation of boundaryvalue problems. Kiev: "Naukova Dumka", 1986, in Russian, with an English summary, edited and with a preface by Yu. A. Mitropolskii.

[29] A. M. Samoilenko and N. I. Ronto, Numerical-analytic methods in the theory of boundary-value problems for ordinary differential equations. Kiev: "Naukova Dumka", 1992, in Russian, edited and with a preface by Yu. A. Mitropolskii.

Authors' addresses

\section{A. Rontó}

Institute of Mathematics, Academy of Sciences of Czech Republic, Žižkova 22, 61662 Brno, Czech Republic

E-mail address: ronto@math.cas.cz

\section{Rontó}

Department of Analysis, University of Miskolc, 3515 Miskolc-Egyetemváros, Hungary

E-mail address: matronto@gold.uni-miskolc.hu 